\title{
L-Selektin ist kein zuverlässiger Marker
}

Fragestellung: Die Studie untersuchte, ob bei Patienten mit Multipler Sklerose (MS) die Bestimmung von L-Selektin (CD62L) als Biomarker für ein erhöhtes Risiko herangezogen werden könnte, im Verlauf einer Therapie mit Natalizumab an einer progressiven multifokalen Leukenzephalopathie (PML) zu erkranken.

Hintergrund: Die Behandlung der MS mit Natalizumab ist mit der seltenen Entwicklung einer PML assoziiert. Bereits vor drei Jahren hatten sich Hinweise darauf ergeben, dass die Messung von CD62L auf zuvor kryokonservierten CD4+-T-Zellen eine Möglichkeit darstellen könnte, Patienten mit erhöhtem PMLRisiko frühzeitig zu erken-

Lieberman LA, Zeng, W, Singh $C$ et al. CD62L is not a reliable biomarker for predicting PML risk in natalizumab-treated R-MS patients. Neurology 2016; 86: $375-81$ nen [1]. Die Messung von CD62L ist jedoch methodisch anspruchsvoll und Veränderungen in der Methodik oder kompromittierte Biomaterialien können zu falschen Ergebnissen führen.
Patienten und Methodik: Für die vorliegende Studie wurden Proben von Patienten aus drei verschiedenen klinischen Studien verwendet (STRATA, STRATIFY-2, und Genetics). Aus der STRATA-Studie waren Proben von neun Patienten vor der Entwicklung der PML verfügbar. Diese wurden fluoreszenzmarkiert, fixiert und anschließend durchflusszytometrisch analysiert.

Ergebnisse: Die in dieser Studie gemessenen CD62L-Werte hingen mit der Viabilität der Zellen nach dem Auftauen zusammen und waren sehr variabel im Verlauf der Natalizumabtherapie sowie zwischen den Patienten. Es konnte kein Schwellenwert berechnet werden, der ein höheres PML-Risiko andeuten würde.

Schlussfolgerungen: Die Bestimmung von CD62L ist mit der in dieser Studie angewendeten Methodik in den analysierten Biomaterialien kein Marker für ein erhöhtes PML-Risiko und nur schwer auf Zellen zu detektieren, deren Viabilität beeinträchtigt wurde.

\section{- Kommentar von Nicholas Schwab und Heinz Wiendl, Münster}

\section{Eine Empfehlung kann aufgrund dieser Daten nicht gegeben werden}

Die Ergebnisse stehen im Gegensatz zu den bisherigen Veröffentlichungen [2, 3], die zeigen konnten, dass niedrige CD62LWerte auf ein erhöhtes PML-Risiko hinweisen. In dieser Studie zeigten sich einige wichtige methodologische Probleme:

- Eine stabile CD62L-Messung ist abhängig von der Qualität der verwendeten Biomaterialien, was seit langem bekannt ist und auch in dieser Studie bestätigt wird. Die verwendeten Blutproben waren kompromittiert durch verschiedene Faktoren (z. B. wurden die Blutzellen erst nach bis zu 30 Stunden Verschickung isoliert und anschließend bis zu vier Jahre gelagert). Das führt in der Studie zu im internationalen Vergleich sehr niedrigen CD62L-Werten, wodurch eine Aussage über pathologisch „erniedrigte“ Werte in PML-Risikopatienten erschwert wird.

- Die Anzahl an Patienten ist nicht hoch genug für eine Aussage, ob CD62L als Biomarker funktioniert oder nicht. Vorherige Arbeiten [2] schlossen 1.410 Patienten in die Studie ein.

- Die vorher publizierte Messmethodik wurde verändert, um so einen potenziellen globalen Einsatz der Technik zu ermöglichen. Es wurde stabilisierendes Agens (Benzonase) verwendet und die Zellen wurden vor der Messung fixiert. Es besteht die Möglichkeit, dass diese Änderungen dazu führten, dass die Studie fehlschlug, indem die zu detektierende Fragilität der Zellen chemisch stabilisiert wurde.

- Die Autoren vergleichen Gruppen von Patientenproben anstatt Patienten. Dadurch werden die Statistiken unzulässig beeinflusst, je nach dem wie viele Proben von einem Patienten in die Studie einflossen.
Diese retrospektive Studie ersetzt keine kontrollierte prospektive Evaluierung des Biomarkers und folglich kann aufgrund der Ergebnisse keine Empfehlung gegeben werden.

Der Biomarker wurde 2013 beschrieben [1], 2015 validiert [2], und von einer unabhängigen Gruppe reproduziert [3]. Weiterhin wurde die biologische Grundlage des Biomarkers untersucht [4] und Kongressbeiträge vorgestellt, die den Marker unterstützen [5]. Die vorliegende Arbeit stellt den ersten negativen Bericht dar, mit den oben beschriebenen Caveats. Der Marker wird derzeit im deutschsprachigen Raum eingesetzt.
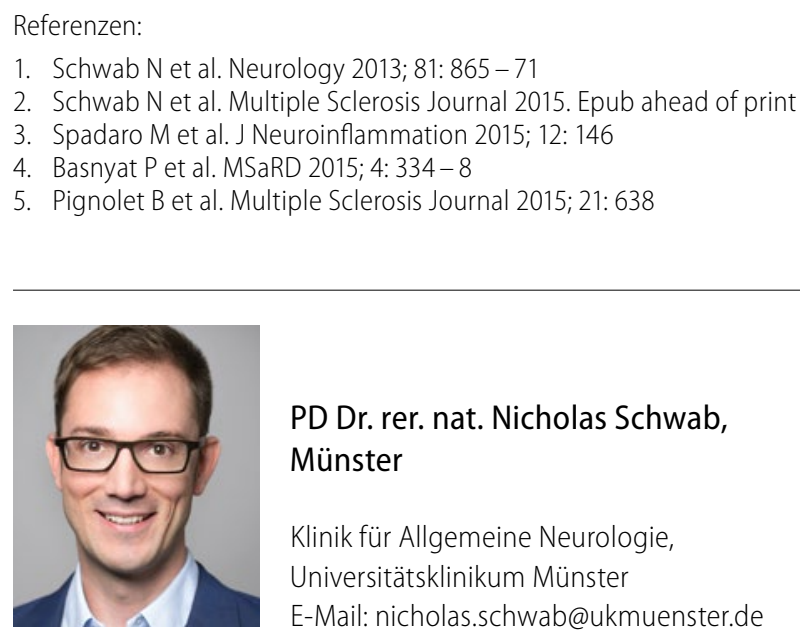

PD Dr. rer. nat. Nicholas Schwab, Münster

Klinik für Allgemeine Neurologie, Universitätsklinikum Münster E-Mail: nicholas.schwab@ukmuenster.de 